\title{
The effect of a stimulation drink on metabolic rate and haemodynamic parameters in comparison to a caffeinated placebo: a randomised, crossover, double blinded study
}

\author{
H. J. Dady and Y. Mavrommatis \\ Dietetics Group, Faculty of Life Sciences, London Metropolitan University, 166-220 Holloway Road, \\ London, $N 7$ 8DB, UK
}

Marketing and consumption of stimulation (or energy) drinks (SDS), has increased considerably in the last two decades ${ }^{(1,2)}$. Claims made regarding such drinks include that they encourage weight loss by increasing energy expenditure (EE) and metabolic rate ${ }^{(1)}$. Some studies have found a significant acute increase in resting $\mathrm{EE}$ at one, two and three hours following ingestion of an $\mathrm{SD}^{(2,3)}$, however these studies have used very high caffeine products (200-300 mg), with a low or no caffeine placebo. It is therefore unclear whether this increase is due to the high caffeine content of the SDS tested, or their specific blend of ingredients, as suggested by marketing campaigns ${ }^{(4)}$. In addition, studies have raised health concerns regarding the effects of SDS on haemodynamic function ${ }^{(4)}$.

This study aimed to explore whether an SD would increase resting EE, heart rate (HR) and blood pressure (BP), in comparison to a caffeinated placebo and baseline measurement. A sample of 11 healthy adult volunteers participated. For each, resting EE was measured using a GEM indirect calorimeter on three occasions. The first was a baseline measurement and on the other two occasions participants ingested either an SD (KX Sugar Free Stimulation Drink) or a placebo (PB: Diet Coke Citrus Zest) two hours before measurement. Both beverages were matched for caffeine content $(80 \mathrm{mg})$ and volume. Blood pressure and HR were also measured on each occasion, and measurement conditions were standardised according to best practice methods to promote reliable measures ${ }^{(5)}$. The table shows mean EE, diastolic and systolic BP and HR under each condition.

\begin{tabular}{|c|c|c|c|c|c|c|c|}
\hline & \multirow[b]{2}{*}{$n$} & \multicolumn{2}{|c|}{ Baseline } & \multicolumn{2}{|c|}{ Stimulation drink } & \multicolumn{2}{|c|}{ Placebo } \\
\hline & & Mean & SD & Mean & SD & Mean & SD \\
\hline Energy expenditure (Kcal/min) & 11 & 1.28 & 0.16 & 1.33 & 0.23 & 1.36 & 0.18 \\
\hline Systolic BP (mm Hg) & 11 & 120.3 & 8.6 & 120.4 & 11.1 & 119.5 & 10.2 \\
\hline Diastolic BP (mm Hg) & 11 & 75.2 & 10.4 & 75.6 & 6.8 & 73.4 & 8.4 \\
\hline Heart rate (bpm) & 11 & 70.2 & 13.3 & 68.6 & 14.1 & 68.1 & 9.3 \\
\hline
\end{tabular}

Values are means at baseline and two hours following consumption of a stimulation drink or caffeinated placebo. For all variables, no significant differences between conditions were found (Friedman's ANOVA): $p>0.05$ ).

Participants' mean EE did not significantly change between baseline and two hours following consumption of the SD or placebo drink. Similarly, no significant differences were found for HR or BP between conditions. The findings of this study indicate that sDs containing a moderate level of caffeine do not significantly increase resting EE, and therefore are unlikely to function well as a weight loss aid. In addition, the significant increase in resting EE following ingestion of an SD observed in previous studies ${ }^{(2,3)}$ is likely to be due to the very high caffeine content contained in the products tested, rather than any other ingredients. The finding that BP and HR were not significantly increased two hours following consumption of the SD is consistent with previous studies ${ }^{(3,6)}$.

1. Reissig CJ, Strain EC \& Griffiths RR (2009) Caffeinated energy drinks - a growing problem. Drug Alcohol Depend 99(1-3): 1-10.

2. Dalbo VJ, Roberts MD, Stout JR et al. (2008) Acute effects of ingesting a commercial thermogenic drink on changes in energy expenditure and markers of lipolysis. J Int Soc Sports Nutr 20(5): 6.

3. Wilborn C, Taylor L, Poole C et al. (2009) Effects of ingesting a commercial thermogenic product on hemodynamic function and energy expenditure at rest in males and females. Appl Physiol Nutr Metab 34(6): 1073-8.

4. Higgins JP, Tuttle TD \& Higgins CL (2010) Energy beverages: content and safety. Mayo Clin Proc 85(11): 1033-41.

5. Compher C, Frankenfield D, Keim N et al. (2006) Best practice methods to apply to measurement of resting metabolic rate in adults: A systematic review. J Am Diet Assoc 106, 881-903.

6. Rudelle S, Ferruzzi MG, Cristiani I et al. (2007) Effect of a thermogenic beverage on 24-hour energy metabolism in humans. Obesity (Silver Spring) 15(2): $349-55$. 